\title{
A case-control investigation of the relation between hyperlipidaemia and calcific aortic valve stenosis
}

P T Wilmshurst, R N Stevenson, H Griffiths, J R Lord

\begin{abstract}
Objective-To investigate the relation of hyperlipidaemia to calcific aortic valve stenosis.

Design-A case-control study designed to detect a clinically relevant difference in the fasting plasma concentrations of total cholesterol between the groups at the 5\% level with a power of $90 \%$. Predefined subgroup analyses were based on presence of significant coronary disease and valve morphology (that is, bicuspid or tricuspid).

Setting-A district general hospital.

Subjects-20 patients with severe calcific aortic stenosis and 20 controls.

Results-Mean (SD) fasting plasma total cholesterol in patients with aortic stenosis was $0.79(1.50) \mathrm{mmol} / 1$ greater than in the controls $(p=0.029)$. The magnitude of differences between patients with aortic stenosis and controls was similar whether the patients had coronary artery disease (0.78 (1.73) $\mathrm{mmol} / \mathrm{l})$ or not $(0.80(1.37)$ mmol/1). The presence of a stenosed tricuspid aortic valve was associated with a significant increase in plasma cholesterol $(1.70(0.87) \mathrm{mmol} / \mathrm{l}, \mathrm{p}=0.012)$. For bicuspid valves the degree of elevation of plasma cholesterol was less and not statistically significant.

Conclusions-Calcific aortic stenosis is associated with hypercholesterolaemia, especially when the valve is tricuspid. Further studies are necessary to confirm that the relation is causal. This finding may have implications for measures to prevent the most common cause of cardiac valve replacement in the developed world.

(Heart 1997;78:475-479)
\end{abstract}

Keywords: calcific aortic stenosis; hypercholesterolaemia; hyperlipidaemia

Aortic valve stenosis is the commonest reason for cardiac valve replacement in the developed world. In the United Kingdom over half of all valve replacements are for this reason. The great majority of cases of aortic stenosis are the result of calcific valve degeneration. If the causes of the degenerative process were understood and if effective preventive measures were employed the clinical benefits to individual patients and financial gains for health care providers would be enormous.
Individuals with bicuspid aortic valves, which are present in about $1 \%$ of the normal population, are excessively represented among patients with calcific aortic stenosis and they have a younger mean age at the time of valve replacement than patients with stenosed tricuspid aortic valves. ${ }^{12}$ This suggests that bicuspid valves are more susceptible to the degenerative process than tricuspid valves. The histological features of stenosis of both bicuspid and tricuspid valves include calcification, fibrosis, and lipid deposition with morphological similarities to atherosclerosis. ${ }^{3}$ A meta-analysis of 33 studies showed a $37 \%$ prevalence of coronary artery disease in patients with calcific aortic stenosis. ${ }^{4}$ This greater than anticipated association suggests the possibility of common aetiological factors. ${ }^{4}$ This is supported by observation that the rate of progression of aortic stenosis on serial Doppler echocardiograms is faster in those patients with coronary artery disease. ${ }^{5}$ The need for coronary grafting at the time of valve replacement is greater in patients with a tricuspid valve, ${ }^{2}$ which suggests that atherogenic factors may have a more important role in aortic valve stenosis when the valve is tricuspid.

In order to determine whether a relation exists between calcific aortic stenosis and hypercholesterolaemia we performed a casecontrol study. We compared fasting plasma concentrations of lipids in patients with significant aortic stenosis, defined by the need for valve replacement, and population controls. The aim of the study was to determine whether there were clinically relevant differences between the fasting plasma concentrations of cholesterol and triglyceride in the groups.

\section{Methods}

PATIENTS

Patients with calcific aortic stenosis were included if the degree of stenosis measured during cardiac catheterisation was severe enough for valve replacement to be indicated. In our department the decision to recommend surgery was based on gradient across the aortic valve in patients with good left ventricular function, and on the Gorlin formula - which uses both valve gradient and flow-in patients with impaired ventricular contraction. ${ }^{6}$ The presence of valve calcification was determined by fluoroscopy. Patients with haemodynamically important aortic regurgitation or mitral valve disease were excluded. We considered that those with mitral valve disease would 
probably have rheumatic heart disease, which would introduce a confounding factor into the analysis. Patients were neither included nor excluded by the presence of significant coronary artery disease, defined as more than $50 \%$ narrowing in one or more coronary arteries on coronary angiography.

CONTROLS

The control subjects were taken from the computer database of a large local group general practice. For each patient with aortic stenosis, all the individuals of the same gender and same age in years were identified by birth date (in order to prevent bias arising from recognition of individuals) and one was chosen at random as the control. The only exclusion criterion for controls was aortic stenosis, determined by clinical examination by a cardiologist and confirmed by Doppler echocardiographic demonstration of an aortic valve gradient in those with a murmur. Control subjects were not excluded by the presence of indicators of hyperlipidaemia or aortic sclerosis, defined as valve thickening without stenosis.

All subjects gave informed written consent to the study, which was approved by the hospital ethics committee.

\section{LABORATORY ANALYSES}

In each case venous blood was sampled after a 12 hour overnight fast, and plasma concentrations of total cholesterol and triglyceride were measured by standard enzymatic methods using the Beckman CX7 analyser (Beckman Instruments, Brea, California, USA). The between batch coefficient of variation in our laboratory is $1.6 \%$ for cholesterol and $2.8 \%$ for triglyceride.

\section{STATISTICAL ANALYSIS}

A large difference in plasma concentrations of lipids between patients with aortic stenosis and the rest of the population would be very obvious and a small difference would be clinically irrelevant. A difference between the groups of two or three times the combined analytical and biological coefficient of variation for cholesterol in our populationapproximately 0.7 to $1.05 \mathrm{mmol} / 1$ cholesterolwould be clinically relevant but not immediately obvious. (The combined analytical and biological coefficient of variation for cholesterol is approximately $6 \%$ and the mean plasma cholesterol concentration in normal elderly people in our district is approximately 6 $\mathrm{mmol} / \mathrm{l}$.) We calculated that 20 patients with aortic stenosis and 20 case controls would be sufficient to detect at the $5 \%$ level a clinically relevant difference in fasting plasma concentration of total cholesterol $(0.7$ to $1.05 \mathrm{mmol} / \mathrm{l})$ between the groups, with a power of $90 \%$. Predefined subgroup analyses were based on the presence of significant coronary artery disease and on aortic valve morphology (that is, determination of whether the valve was bicuspid or tricuspid by assessment at the time of valve replacement). Comparisons between the groups was with the paired $t$ test (two tailed). A $\mathrm{p}$ value $<0.05$ was considered significant.
Results are expressed as mean (SD) or range as appropriate.

\section{Results}

Each group consisted of nine men (mean age 65.4 , range 50 to 70 years) and 11 women (mean age 66.4, range 56 to 75 years).

There was heavy calcification of the aortic valve in each of the subjects with aortic stenosis, and the mean value of peak aortic valve gradient at the time of cardiac catheterisation was 74.5 (range 50 to 135 ) $\mathrm{mm} \mathrm{Hg}$. Nine patients (four men and five women, mean age 64.6 , range 50 to 78 years) with aortic stenosis had significant coronary artery disease (four single, two double, and three triple vessel disease) and coronary bypass graft surgery was planned at the time of valve replacement in each. Eleven patients (mean age 67.1, range 51 to 75 years) did not have significant coronary artery disease (nine with absolutely normal coronary arteries on angiography and two with minor irregularities), and in none of these was coronary grafting performed. Two patients did not have the planned surgery, including the one with the greatest valve gradient. The reasons were patient preference in one case and the concerns of the anaesthetist about coexistent lung disease in the other. Valve morphology could not be determined with certainty in these two cases and in seven other cases the surgeons were either uncertain or failed to record the valve morphology. In the remaining cases, six (mean age 64.2, range 51 to 75 years) had bicuspid valves and five (mean age 66.2, range 56 to 75 years) had tricuspid valves.

Three control subjects had systolic murmurs not associated with aortic stenosis. In one case the murmur was maximal in the mitral area and echocardiography showed mild mitral regurgitation and no other abnormality. In two controls the murmurs were maximal in the aortic area. Echocardiography in one showed nodular thickening of the aortic valve without evidence of stenosis. The other had a normal echocardiogram. These three individuals were included as controls because they did not have predefined exclusions.

Eight controls had findings which suggested the possibility of hyperlipidaemia. One was an insulin dependent diabetic, one had a history of amaurosis fugax (without murmurs or bruits), one had a subclavian bruit without symptoms, one had a history of myocardial infarction, one had polycystic kidneys with hypertension, and three had essential hypertension.

Both groups were taking a variety of medications (mean of 2.8 drugs per patient with aortic stenosis and 2.3 drugs per control subject) from 32 classes of drugs. The drugs most often taken were non-steroidal anti-inflammatory drugs (including low dose aspirin), paracetamol and other analgesics, and treatments for dyspepsia. There was no significant difference between the groups in medication taken including cardiac drugs; loop diuretics (five aortic stenosis $v$ two controls), thiazide diuretics (three $v$ three), angiotensin converting enzyme inhibitors (one $v$ two), $\beta$ adrenergic blockers (five $v$ four), class 1 antiarrhythmic 
Table 1 Fasting plasma concentrations of lipids

\begin{tabular}{|c|c|c|c|c|c|}
\hline & $\begin{array}{l}\text { Aortic stenosis } \\
(\mathrm{mmol} / \mathrm{l})\end{array}$ & $\begin{array}{l}\text { Case controls } \\
\text { (mmol/l) }\end{array}$ & $\begin{array}{l}\text { Mean difference } \\
(\mathrm{mmol} / \mathrm{l})\end{array}$ & $\begin{array}{l}\text { Number in } \\
\text { each group }\end{array}$ & p Value \\
\hline \multicolumn{6}{|l|}{ Cholesterol } \\
\hline All AS & $6.90(1.33)$ & $6.11(1.24)$ & $0.79(1.50)$ & 20 & $0.029^{\star}$ \\
\hline $\mathrm{AS}+\mathrm{CAD}$ & $7.21(1.32)$ & $6.43(1.47)$ & $0.78(1.73)$ & 9 & 0.21 \\
\hline AS, no CAD & $6.65(1.34)$ & $5.85(1.02)$ & $0.80(1.37)$ & 11 & 0.082 \\
\hline AS bicuspid & $6.88(1.49)$ & $5.90(1.42)$ & $0.98(1.34)$ & 6 & 0.13 \\
\hline AS tricuspid & $7.88(0.64)$ & $6.18(0.64)$ & $1.70(0.87)$ & 5 & $0.012^{\star}$ \\
\hline \multicolumn{6}{|l|}{ Controls, no } \\
\hline murmur & $6.78(1.26)$ & $5.72(0.85)$ & $1.06(1.20)$ & 17 & $0.002^{\star}$ \\
\hline \multicolumn{6}{|l|}{ Triglyceride } \\
\hline All AS & $1.61(0.77)$ & $1.68(0.90)$ & $-0.07(1.20)$ & 20 & 0.79 \\
\hline $\mathrm{AS}+\mathrm{CAD}$ & $1.77(0.81)$ & $1.92(1.05)$ & $-0.15(1.58)$ & 9 & 0.78 \\
\hline AS, no CAD & $1.48(0.74)$ & $1.48(0.76)$ & $-0.01(0.84)$ & 11 & 0.99 \\
\hline AS bicuspid & $1.23(0.37)$ & $1.18(0.41)$ & $0.04(0.23)$ & 6 & 0.67 \\
\hline AS tricuspid & $2.40(0.80)$ & $1.40(0.80)$ & $1.00(0.99)$ & 5 & 0.09 \\
\hline \multicolumn{6}{|l|}{ Controls, no } \\
\hline murmur & $1.70(0.80)$ & $1.61(0.87)$ & $0.09(1.18)$ & 17 & 0.77 \\
\hline
\end{tabular}

*Statistically significant.

AS, aortic stenosis; CAD, coronary artery disease.

drugs (one $v$ three), amiodarone (one $v$ none), dihydropyridines (four $v$ two), or warfarin (one $v$ none). No subject in either group was taking lipid lowering drugs.

The results of lipid analysis in the patients with aortic stenosis and the controls, and the subgroup analysis, are shown in table 1 . Seventeen of the patients with aortic stenosis had a fasting plasma concentration of cholesterol that was greater than their respective controls. The mean fasting plasma concentration of total cholesterol in the patients with aortic stenosis was higher than in the controls $(p=0.029$, difference $=0.79(1.50) \mathrm{mmol} / \mathrm{l})$. The mean fasting plasma concentration of total cholesterol in our control subjects $(6.11 \mathrm{mmol} / \mathrm{l})$ was similar to the anticipated mean concentration for normal elderly individuals in our district (about 6 $\mathrm{mmol} / \mathrm{l})$.

Subgroup analysis showed that the differences in cholesterol concentrations between the patients with aortic stenosis and their controls were similar whether those with aortic stenosis had coronary artery disease $(0.78$ (1.73) $\mathrm{mmol} / \mathrm{l})$ or not $(0.80(1.37) \mathrm{mmol} / \mathrm{l})$. These differences did not achieve statistical significance at the 5\% level, which may be related to the small sizes of subgroups. In patients with aortic stenosis, symptoms were angina in six of nine with coronary artery disease and six of 11 without coronary disease. A further two in the later group had atypical chest pain. All 14 had dyspnoea on exertion, as did three without pain. The remaining three, who all had coronary disease, had no pain or dyspnoea but complained of syncope, dizziness, or palpitations. Chest pain was cured by surgery in all cases-by valve replacement alone in those patients without coronary disease.

The fasting plasma concentration of cholesterol in patients known to have stenosed tricuspid aortic valves was $1.70(0.87) \mathrm{mmol} / \mathrm{l}$ higher than in their controls $(p=0.012)$. In patients known to have bicuspid aortic valves the degree of elevation of mean fasting plasma cholesterol concentration compared with their controls was less and not significant.
Of the control subjects, the three with murmurs had the greatest fasting plasma concentrations of cholesterol. If comparisons were performed after excluding these subjects from the analysis, the mean difference in fasting plasma cholesterol between the patients with aortic stenosis and their controls $(\mathrm{n}=17)$ increased to $1.06(1.20) \mathrm{mmol} / \mathrm{l}(\mathrm{p}=0.002)$.

There was no significant difference in plasma triglyceride concentrations between patients with aortic stenosis and their controls, for either the whole groups or the subgroups.

\section{Discussion}

With these data we have shown a clinically relevant association between aortic stenosis and hypercholesterolaemia. This association may be more important when the valve is tricuspid. However, an important consideration must be whether the presence of concomitant coronary disease in some patients may have produced symptoms, particularly angina, which prompted investigation and hence resulted in bias towards finding an association between aortic stenosis and hypercholesterolaemia.

Exclusion of patients with coronary artery disease could itself result in bias. Calcific aortic stenosis is multifactorial, or at least bifactorial, because demographic characteristics of patients with stenosed bicuspid and tricuspid valves differ. ${ }^{2}$ Calcific aortic stenosis is commonly associated with coronary disease. In a meta-analysis of 33 studies reported between 1968 and 1992, significant coronary artery disease was present in 1302 of 3509 patients with aortic stenosis (37\%). ${ }^{4}$ This figure is not dissimilar to the $45 \%$ prevalence of coronary artery disease in patients with aortic stenosis in our study. Coronary disease is more common in patients with tricuspid valves. ${ }^{2}$ Thus in a study in which only patients with aortic stenosis and normal coronaries were studied a false negative result might be produced by excluding patients with stenosis of the tricuspid valves, who a priori might be expected to be the ones most likely to have hypercholesterolaemia.

Exclusion of patients with symptoms that may result from coronary disease introduces other biases. Angina is of course a symptom of both aortic stenosis and coronary disease. In our patients this symptom was equally common in those with and without coronary artery disease. Every patient studied had symptoms common to both aortic stenosis and coronary disease. If only patients with significant aortic stenosis but without coronary disease or coronary symptoms were studied, the group would be so atypical of patients with aortic stenosis that the findings might have little clinical relevance.

Our data clearly showed that the inclusion of nine patients with coronary disease and aortic stenosis did not result in bias. Eight of 20 controls also had conditions which are associated with hyperlipidaemia, including a history of myocardial infarction, diabetes mellitus, amaurosis fugax, vascular disease, polycystic kidney disease, and hypertension. Our subgroup analysis showed that inclusion of cases with coronary disease did not influence the overall 
findings. We found that for the whole group of patients, mean cholesterol was 0.79 (1.50) $\mathrm{mmol} / \mathrm{l}$ higher in those with aortic stenosis than in the controls $(\mathrm{p}=0.029, \mathrm{n}=20)$. In those with aortic stenosis and coronary disease the mean cholesterol was 0.78 (1.73) $\mathrm{mmol} / 1$ higher than in their controls $(\mathrm{p}=0.21, \mathrm{n}=9)$ and in those without coronary disease it was $0.80(1.37) \mathrm{mmol} / 1$ higher $(\mathrm{p}=0.082, \mathrm{n}=11)$. In other words, in both subgroups the findings were almost identical to the whole.

The results of subgroup analysis might have been more impressive if we had studied more patients, but there are two reasons why we did not. First, there is an ethical imperative in prospective research to perform a power calculation before the start of a study and then to study only the number of patients required to address the primary aim in order to avoid subjecting extra subjects unnecessarily to inconvenience, pain, or risk. This imperative also avoids wasting resources by preventing investigators performing more tests than the minimum number required. In addition, if we had needed to study large numbers to show a significant difference this would suggest that the effect was not clinically important.

These data alone do not prove a causal relation, but in combination with the findings of others, ${ }^{457}$ they suggest that hypercholesterolaemia may be an aetiological factor in the development of aortic stenosis. Our findings suggest that hypercholesterolaemia may be more important for development of aortic stenosis when the valve morphology is tricuspid. This is supported by the observation that coronary artery disease requiring coronary grafting is more commonly associated with aortic stenosis when the valve is tricuspid. ${ }^{27}$

Two previous case-control studies have sought a relation between aortic stenosis and hypercholesterolaemia, but have produced conflicting results. ${ }^{89}$ In each of those studies the degree of aortic stenosis was not severe enough in all patients to require valve replacement. The controls in both studies were hospital patients, including those undergoing cardiac catheterisation for reasons other than aortic stenosis-including severe coronary artery disease and unstable angina, and surgical patients - rather than random population controls. One study failed to show an association between aortic stenosis and hypercholesterolaemia but was biased by exclusion of patients with aortic stenosis who had coronary artery disease, yet allowed inclusion of controls with coronary disease. ${ }^{9}$ The other study had a remarkably low proportion $(6 \%)$ of subjects who had a definitely bicuspid valve. ${ }^{8}$ Both studies used $\chi^{2}$ tests to compare the subjects and controls but used different and arbitrary cholesterol concentrations to subdivide their groups. Therefore the differing conclusions of these two studies may have been the result of their design. The results obtained were certainly dependent on choice of cut offs defining hypercholesterolaemia. This difficulty was eliminated in our study by use of absolute cholesterol concentrations.
A recent report supports the role of hypercholesterolaemia in the aetiology of calcific aortic stenosis. Rallidis and colleagues reported a case of calcific aortic stenosis in a 25 year old woman with homozygous hypercholesterolaemia and stated that they had seen five similar cases (age 14 to 31 years). ${ }^{10}$ If a severe rise in plasma cholesterol concentration, to the degree associated with homozygous hypercholesterolaemia, for two or three decades can produce aortic stenosis in some but not all patients, it is possible that milder degrees of cholesterol elevation for longer periods might also cause aortic stenosis. Demonstration of a modest degree of hypercholesterolaemia on a single measurement does not prove that an individual has sustained hypercholesterolaemia, but prospective data from large coronary risk analysis studies has shown that (off treatment and remote from events known to affect lipid profiles) the plasma cholesterol concentration remains constant over many years relative to the concentrations in peers matched for age and gender. ${ }^{11}{ }^{12}$ If this were not so there would be no rationale for lipid reduction to modify coronary risk.

We must also consider whether it is plausible that the degree of elevation of plasma cholesterol concentration in our patients, if sustained, would be sufficient to produce valve stenosis. For the whole group, plasma cholesterol concentration was 0.79 (1.50) $\mathrm{mmol} / \mathrm{l}$ higher than in the controls, and for those with stenosis of definitely tricuspid valves the cholesterol concentration was $1.70(0.87)$ $\mathrm{mmol} / \mathrm{l}$ higher $(\mathrm{p}=0.012)$. We do not know whether this degree of hypercholesterolaemia, if sustained for a number of decades, would have been sufficient to cause aortic stenosis. The average age of subjects was 66 years. Could such an elevation of plasma cholesterol concentrations for such a period have a clinically important effect on a heart valve? We can draw comparisons from studies of hypercholesterolaemia in coronary artery disease, in which similar degrees of hypercholesterolaemia at recruitment were associated with increased risk of coronary disease at follow up. ${ }^{11}{ }^{12}$ In the Scandinavian simvastatin survival study, reducing total cholesterol by just under 1.7 $\mathrm{mmol} / 1$ for on average 5.4 years reduced coronary deaths by $42 \%$ and all cause mortality by $30 \% .^{13}$ In the West of Scotland coronary prevention study, reducing cholesterol by 1.4 $\mathrm{mmol} / 1$ for 4.9 years reduced cardiac deaths by $32 \%$ and all cause mortality by $22 \% .{ }^{14}$ In both trials survival curves for placebo and treated groups started to diverge during the second year. If lowering cholesterol by about 1.5 $\mathrm{mmol} / \mathrm{l}$ can have definite effects on the endothelium lined coronary arteries within five years and possible effects after shorter intervals, it is conceivable that elevation of cholesterol by a similar magnitude for decades might affect endothelium lined cardiac valves. Further, these trials suggest that if our observations are extended and confirm a causal relation, then the degree of cholesterol reduction one might be seeking to prevent aortic 
valve stenosis is achievable using currently available treatment.

Proof of a causal relation between hypercholesterolaemia and calcific aortic stenosis could come from demonstration of an effect on incidence or progression of aortic stenosis by lipid reduction in large epidemiological studies. The studies could be designed to answer that specific question, or they might be designed to answer an incidental question, such as the effect of cholesterol reduction on coronary disease progression. ${ }^{14}$ We may also find that if the results of primary intervention studies of hyperlipidaemia are translated into preventive medical practice, the incidence of aortic stenosis is reduced or the age of peak incidence changes, or both.

1 Davies MJ. Pathology of aortic stenosis in the elderly. Cardiol Elderly 1995;3:161-5.

2 Davies MJ, Treasure T, Parker DJ. Demographic characteristics of patients undergoing aortic valve replacement for stenosis: relation to valve morphology. Heart 1996;75:1748 .

3 Otto CM, Kuusisto J, Reichenbach DD, Gown AM, O'Brien KD. Characterization of the early lesions of "degenerative" valvular aortic stenosis. Circulation 1994;90: 844-53.

4 Mautner GC, Roberts WC. Reported frequency of coronary arterial narrowing by angiogram in patients with valvular aortic stenosis. Am f Cardiol 1992;70:539-40.
5 Peter M, Hoffman A, Parker C, Luscher T, Burckhardt D. Progression of aortic stenosis. Role of age and concomitant coronary artery disease. Chest 1993;103:1715-19.

6 Gorlin R, Gorlin G. Hydraulic formula for calculation of area of stenotic mitral valve, other cardiac valves and central circulatory shunts. Am Heart f 1951;41:1-29.

7 Mautner GC, Mautner SL, Cannon RO, Hunsberg SA, Roberts WC. Clinical factors useful in predicting aortic
valve structure in patients $>40$ years of age with isolated valve structure in patients aortic stenosis. Am $\mathcal{F}$ Cardiol 1993;72:194-8.

8 Deutscher S, Rockette HE, Krishnaswami V. Diabetes and hypercholesterolaemia among patients with calcific aortic stenosis. F Chron Dis 1984;37:407-15.

9 Hoagland PM, Cook EF, Flatley M, Walker C, Goldman L. Case-control analysis of risk factors for presence of aortic stenosis in adults (age 50 years or older). Am $\mathcal{F}$ Cardiol 1985;55:744-7.

10 Rallidis L, Nihoyannopoulos P, Thompson GR. Aortic stenosis in homozygous familial hypercholesterolaemia. Heart $1996 ; 76: 84-5$.

11 Kannel WB, Castelli WP, Gordon T, McNamara PM. Serum cholesterol, lipoproteins, and the risk of coronary heart disease: the Framingham Study. Ann Intern Med 1971;74:1-12

12 Kannel WB, Neaton JD, Wentworth D, Thomas HE, Stamler J, Hulley SB, et al. Overall and coronary heart disease mortality rates in relation to major risk factors in 325,348 men screened for the MRFIT. Am Heart 7 1986;112:82536 .

13 Scandinavian Simvastatin Survival Study Group. Randomised trial of cholesterol lowering in 4444 patients with coronary heart disease: the Scandinavian Simvastatin Survival Study. Lancet 1994;344:1383-9.

14 Shepherd J, Cobbe SM, Ford I, Isles CG, Lorimer AR, Macfarlane PW et al. Prevention of coronary heart disease with pravastatin in men with hypercholesterolemia. $N$ Engl f Med 1995;333:1301-7. 